\title{
Archéopages
}

Archéopages

Archéologie et société

46 | 2018

Maisons

\section{La maison et l'habitat à la fin du Moyen Âge en Normandie}

Pour un dialogue entre archéologie et sources écrites

Houses and dwellings at the end of the Middle Ages in Normandy. In favour of a dialogue between archaeology and written sources

La vivienda y el hábitat a fines de la Edad Media en Normandía. Por un diálogo

entre la arqueología y las fuentes escritas

\section{Vincent Carpentier et Christophe Brière}

\section{OpenEdition \\ Journals}

Édition électronique

URL : https://journals.openedition.org/archeopages/4251

DOI : 10.4000/archeopages.4251

ISSN : 2269-9872

Éditeur

INRAP - Institut national de recherches archéologiques préventives

Édition imprimée

Date de publication : 1 décembre 2018

Pagination : 70-79

ISSN : 1622-8545

Référence électronique

Vincent Carpentier et Christophe Brière, « La maison et l'habitat à la fin du Moyen Âge en

Normandie ", Archéopages [En ligne], 46 | 2018, mis en ligne le 01 décembre 2020, consulté le 03 juin

2021. URL : http://journals.openedition.org/archeopages/4251 ; DOI : https://doi.org/10.4000/

archeopages. 4251 


\title{
La maison et l'habitat à la fin du Moyen Âge en Normandie Pour un dialogue entre archéologie et sources écrites
}

\author{
Vincent Carpentier Inrap, UмR 6273, « Centre Michel de Boüard " \\ Christophe Brière Enseignant
}

Depuis la genèse de l'archéologie des « villages désertés » (École pratique des Hautes Études, 1970), les chercheurs normands ont exhumé un corpus de sites qui permet aujourd'hui de dépasser le cadre étroit de l'« architecture traditionnelle», longtemps cantonnée à l'étude du bâti moderne ou contemporain (Lassure, 1985). Les premières fouilles sur ce thème sont inaugurées dès les années 1970 par Michel de Boüard à SaintUrsin-de Courtisigny, hameau déserté de Courseulles-sur-Mer, au nord de Caen, occupé au XIII ${ }^{\mathrm{e}}$-XIV ${ }^{\mathrm{e}}$ siècle ; à partir de 1999, ces recherches sont poursuivies par Claire Hanusse (Hanusse, 2006) [ill. 1]. En 1987, un autre chantier-école de l'université de Caen est ouvert à Grentheville, à l'est de Caen, à l'emplacement du hameau déserté de Trainecourt. Fouillé de 1987 à 1994, ce village, occupé du XIII ${ }^{\mathrm{e}}$ au XVI ${ }^{\mathrm{e}}$ siècle, est à ce jour l'une des plus vastes fouilles d'un seul tenant en Europe (Taupin, 1996 ; Lorren, 1998). À compter des années 1990, l'archéologie préventive intervient à son tour dans la vallée de la Dives, à Vieux-Fumé (Hincker, Thiron, Maneuvrier, 1999), Moult (Carpentier, 2015) ou Jort (Carpentier, 2017). Toutes ces fouilles ont produit un corpus de plans, d'objets, de restes organiques (graines, os) permettant d'asseoir des datations et d'aborder la question de l'économie et du quotidien. Cependant, l'image qu'elles nous renvoient des réalités du monde paysan est souvent lacunaire (Lorren, 2002). Il est, de fait, nécessaire de les enrichir, ce qu'il est possible de faire à la lumière de sources écrites à ce jour sous-exploitées par les archéologues. Quelques exemples précis empruntés à la vallée de la Dives, à l'est de Caen, montrent comment un dialogue peut être instauré entre textes et archéologie.
Une mine documentaire inexploitée

Le registre de tabellionnage d'Argences, Troarn et Varaville forme un imposant recueil de 280 folios en parchemin, composé de 1386 à 1417 par Colin du Vernay, tabellion de Caen en charge des sergenteries d'Argences, Troarn et Varaville. Conservé à Caen, aux Archives départementales du Calvados, sous la cote $7 \mathrm{E} 4$, il est à peu près totalement inédit à l'exception de quelques notices paléographiques (Sauvage, 1911, p. XXXVII ; Le Lorier, 1911). Ce registre, qui réunit trois segments respectivement datés d'octobre 1386 à avril 1401, de juin 1408 à février 1409 et d'avril-mai 1417 (n. st.), est l'un des plus précoces de toute la France du nord (Bernard, 1978, p. 121122). En outre, il peut être complété par plusieurs autres sources concernant le même territoire à la même époque, notamment par le fonds de l'abbaye Saint-Martin de Troarn. Rappelons que d'autres registres de tabellions normands inédits nous sont parvenus pour les $\mathrm{XIV}^{\mathrm{e}}-\mathrm{XV}^{\mathrm{e}}$ siècles. Récemment, ces fonds ont suscité un regain d'intérêt parmi les historiens et certains, comme ceux de Lisieux, perdu depuis la seconde guerre mondiale, ont connu une véritable résurrection

(Maneuvrier, 2010).

Pour ne s'en tenir quà lui, le registre de Colin $\mathrm{du}$ Vernay constitue une mine de renseignements sur les rouages du quotidien à la veille de l'occupation anglaise de la Normandie (1417). Officier de l'administration royale, le tabellion, notaire avant la lettre, gère et consigne les actes de la vie courante, baux, ventes, contrats (Angers, 1994). Son registre dépeint un monde paysan dynamique et, entre autres, les formes, modalités de gestion et évolutions qui affectent son habitat. Il est donc possible, à l'instar de ce qu'a accompli 

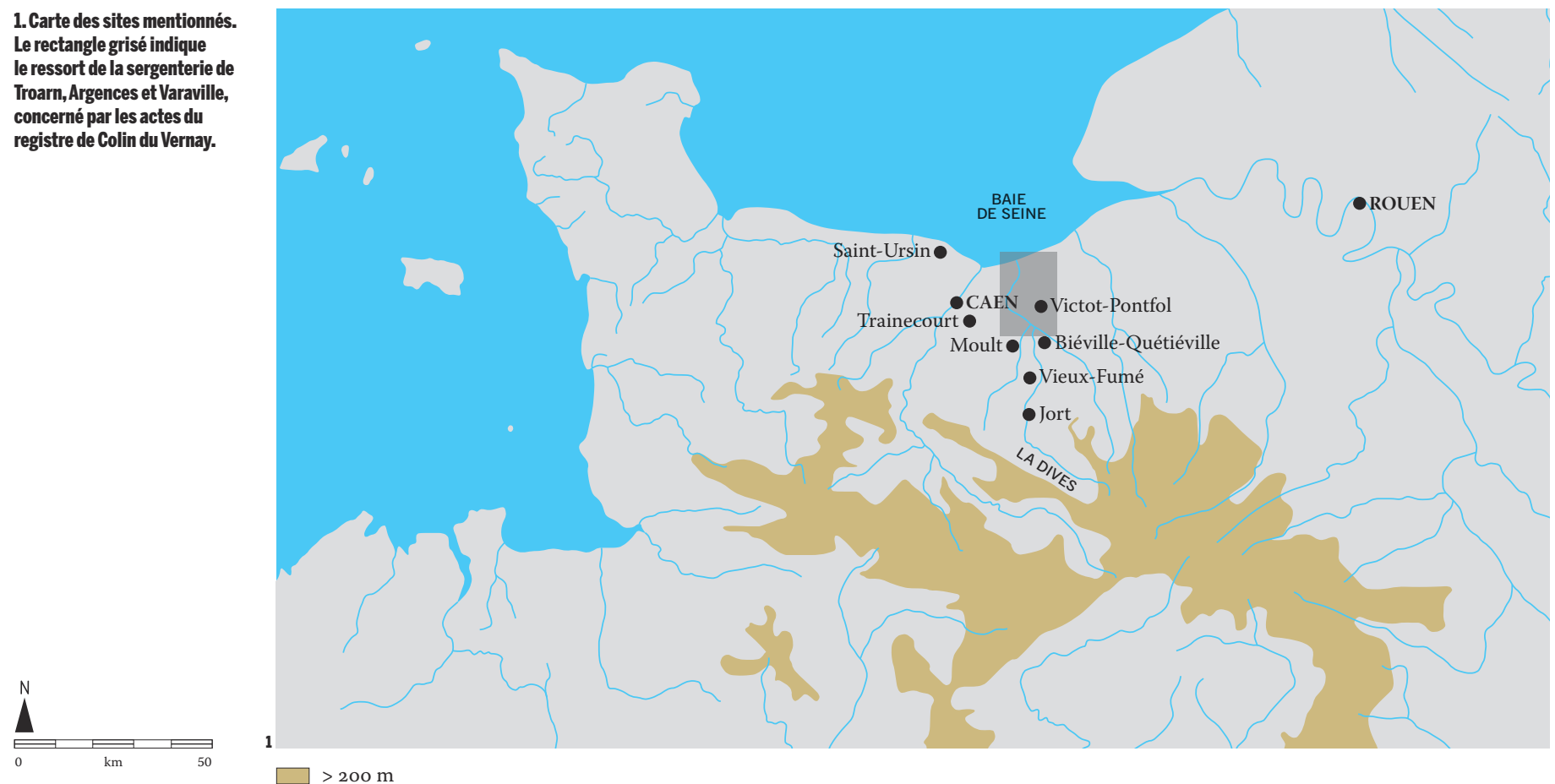

Perrine Mane avec l'iconographie médiévale, d'en esquisser un portrait concret, de type géographique, propre à être confronté aux données archéologiques locales. L'exercice pose évidemment certaines difficultés, à commencer par le danger de vouloir transposer en réalités concrètes le lexique juridique en usage dans les actes notariés (Hanusse, Jarry, 2007). Dans la pratique des tabellions en effet, des termes comme « hébergage », « masnage » ou " masure » désignent non les bâtiments eux-mêmes, mais les différentes formes de baux et de tenures qui encadrent le marché foncier et immobilier à la fin du Moyen Âge. Pour autant, ce lexique n'ôte rien à l'intérêt offert par les multiples mentions d'étages ou d'escaliers, de pièces, de chambres, de dépendances, de voiries, de modes de bâtir et de couvrir dont regorgent les registres. Purement descriptifs, ces détails sont un relais précieux pour l'interprétation et la critique des données de fouille.

\section{L'environnement de la maison}

En premier lieu, la plupart des actes décrivent l'insertion de la maison dans son environnement à travers les bornages, arbres, fossés (le terme désigne une haie sur talus en Normandie), chemins et cours d'eau servant de repères dans le paysage : « pièce de terre (...) à buter sur le gable (pignon) de la maison » (f. 33v); " la moytié d'un herbergage avecquez la maison dessus estante (...) à buter sur le quemin du Roy» (f. 52) ; « une maison avecquez le jardin si comme le tout s'estent jusquez à l'orme et jusquez au périer (orme et poirier), à buter au quemin du roy» (f. 69). Dans les marais, l'eau courante joue clairement à cette époque un rôle attractif pour l'habitat et non l'inverse : ainsi, à Varaville, une maison est mitoyenne d'une sente et d'un « douet » ou ruisseau (f. 43) ; à Troarn, une autre jouxte «le cours de l'eau à buter sur le quemin du Roy» (f. 42). Au sein de cet espace précisément borné et arpenté, la relation est toujours étroite entre la maison et les voies de communication; en témoigne très clairement, au début du XIV siècle, un État des chemins de la baronnie de Troarn, relatif aux droits de police des voies, poids et mesures, de l'abbé de Troarn (Sauvage, 1911, Pr. XXIV). On relève par ailleurs que l'habitat rural s'organise assez largement, du moins dans le secteur considéré, en petits hameaux satellites du centre paroissial, regroupant plusieurs maisons et dépendances : le « hamel de Bleville (...) à buter sur le quemin » (f. 20), la « rue au Moygne », à Moult (f. 26) ou encore le « hamel de Grouchet », à Bavent (f. 43), évoquent les sites de Vieux-Fumé, Jort ou Moult, ainsi que plusieurs hameaux désertés repérés en prospection aérienne à cause de leurs microreliefs, comme Biéville-Quétiéville ou Victot-Pontfol [ill. 2-3].

\section{Les fonctions, formes et matériaux}

La maison est définie dans les actes du tabellion non par son plan mais par ses fonctions : ses habitants doivent pouvoir $\mathrm{y}$ « faire leur lit, mectre leur pot au feu et aller en tous leurs aferes et besoignes » (f. 226v). La maison fournit le coucher, le foyer et l'espace nécessaire au travail quotidien, au logement des animaux ou à la resserre des outils et produits agricoles. Ses dimensions apparaissent rarement dans les actes ; on relève à Janville, en 1400, « le siège d'une maison contenant trente-huit piez de long et trente piez de large » (f. 252), soit environ $12 \times 10 \mathrm{~m}$, ce qui concorde avec de nombreux plans relevés en fouille. À Bréville-les- 

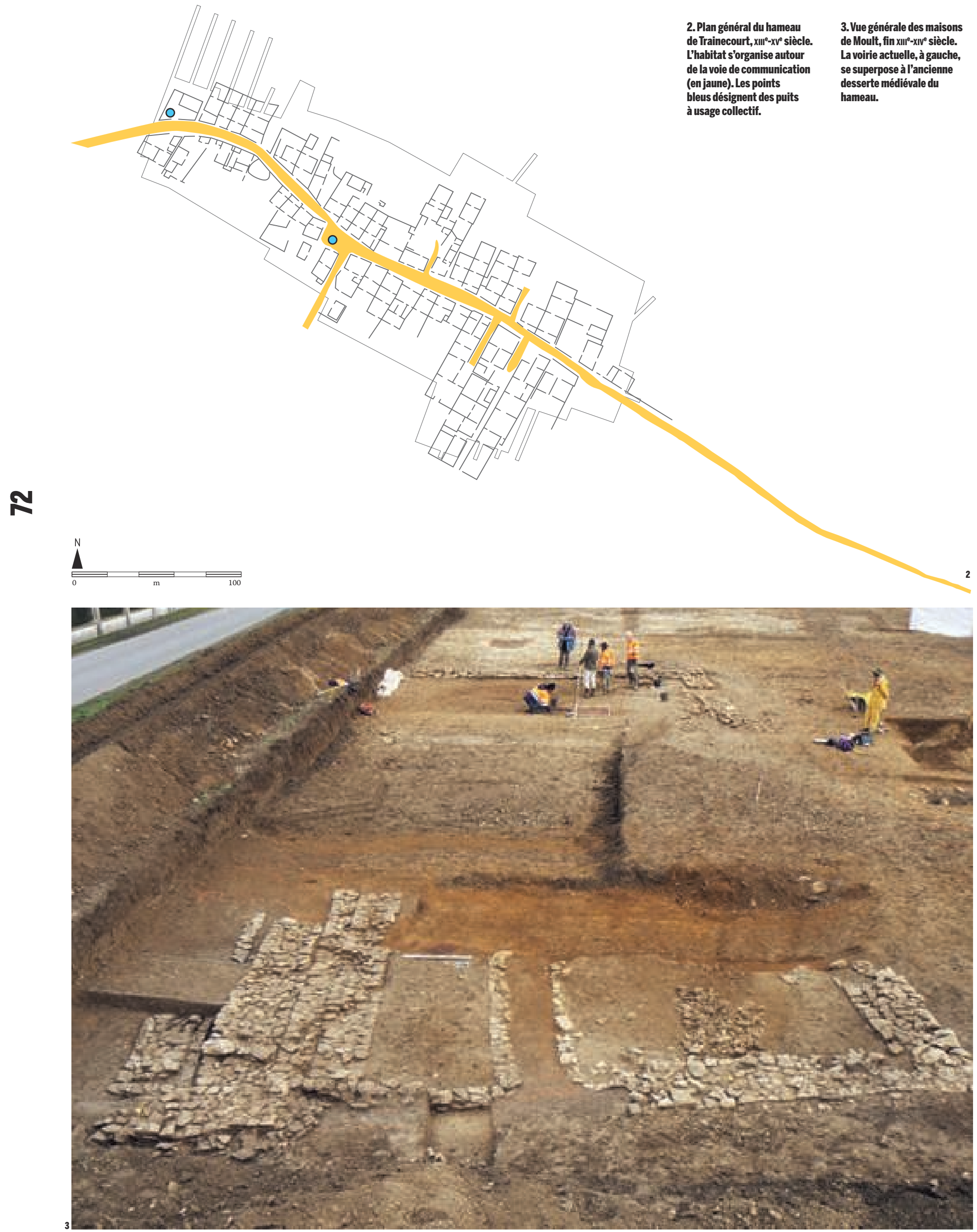
Monts, un acte fait état d'une maison dont le côté est haut de 7 pieds, soit environ $2 \mathrm{~m}$ (f. 65v). On note cependant que les dimensions d'un édifice ne sont pas significatives de sa fonction, et que les textes sont à peu près muets quant aux modes de bâtir. L'archéologie seule a su révéler en Normandie les fondations de pierre sèche des maisons du bas Moyen Âge, y compris dans les secteurs où dominera plus tard le pan-de-bois, de même que les sols décaissés excavés dans le substrat calcaire présents à Trainecourt ou Jort [ill. 4], sans compter les petites constructions semi-enterrées ou sur poteaux.

En revanche, on relève dans l'écrit de très nombreuses mentions de maisons à étage, que les faits archéologiques n'autorisent que très rarement à reconnaître, comme à Moult, sur la foi d'une base supposée d'escalier [ill. 5]. Ces maisons abritent fréquemment sous le même toit des personnes distinctes qui s'en répartissent les étages. Dans ce cas, les actes définissent des droits de passage ou de servitude en rapport avec le partage de la maison entre ses occupants : à Merville, Guillaume Martine et Perrote, sa femme, fieffent « deux estages de maison avecquez son yessue à pié, à cheval et à chareste tout au long du jardin du dit herbergage à Merville, par V sous » (f. 162) ; à Basseneville, Colin Frontin fieffe « deux estages de la maison de haut si comme est assise en la dicte paroisse par devans soleil levant (...) et ledit preneur aira le travers qui est en la dicte maison sans reffus ne contredit » (f. 148v). Plus rares sont les maisons ayant plus de deux niveaux : à Bures-sur-Dives, Guillaume Vigier « quicte à Philippot Vignien, de Bures, une maison à trois estages avecquez le jardin à buter au quemin » (f. 67). Le ou les étages supérieurs abritent généralement des chambres à coucher tandis que le rez-de-chaussée, où se trouve le foyer, est dédié aux pièces à vivre : Durete, veuve Rogier Oton, donne à Jean Guillot tous ses biens et son logis avec jardin à Troarn, en se réservant l'usage du « bas de l'elle (l'aile) (...) et une chambre de haut d'icelle elle avecquez son aler et venir au puis et au jardin du dit herbergage à toutes heures sans reffus ne contredit » (f. 151).

Les modes de couverture font également l'objet de nombreuses mentions ; l'entretien du bâti en général et de la toiture en particulier incombent à l'habitant lui-même : Geoffroy Le Patey, de Robehomme, baille « deux estages de maison avecques le jardin à ce appartenant (...) afin que les dis mariés soient tenus à réfectionner et réparier les dis estages de maison de carpenterie et de couverture et à les tenir en estat » (f. 212v). Ceci fait directement écho aux restes de tuiles, d'ardoises, d'enduits, de petits blocs de pierre taillés présents sur la plupart des sites archéologiques, au sein même de l'habitat ou, du moins, dans les cours attenantes. Dans les secteurs de marais, on utilise ordinairement les roseaux pour les couvertures ou le combustible : à Petiville, le preneur d'une maison en « hébergage » est tenu « à faire couvrir de neusve couverture c'est assavoir de ros et de paiuail (roseau et mélange de terre et de paille) » (f. 64v). On trouve des couvertures en chaume (« glay ») sur les plateaux, comme à Saint-Ursin par exemple (Hanusse, 2006, p. 83), en concomitance avec de l'ardoise verte ou bleue. Bois et bosquets fournissent le matériau nécessaire aux portes et aux clôtures dont les fouilles révèlent assez souvent l'existence sous forme d'alignements de trous de poteaux : Ricart Lennoisie, de Bréville-les-Monts, fieffe « deux estages de maison (...) pour et affin que le dit Adam soit tenu à faire couvrir de glay le costé de la dicte maison par devens soleil levant et à faire faire une closture de bouys et deux us (portes ou portails) l'un dehors et l'autre dedens » (f. 144v). Lorsque la maison en question est d'une certaine envergure, ces travaux en viennent à constituer une charge considérable : à Guillerville, Colin Morant reconnaît avoir pris des religieux de Troarn, pour neuf ans, « un manoir appellé Le Homme avecquez touts ses appartenances et appendances quelconques » pour le prix de 1o livres par an, à charge de « tenir en estat le dit manoir et le coulombier et réparier les fosses et (...) planter le jardin où est le coulombier assis bien et deuement » (f. 99). On reconnaît ici l'un de ces « habitats d'élite», terme consacré de la littérature archéologique, sans qu'il soit pour autant question d'un preneur noble...

\section{Les intérieurs médiévaux}

L'agencement intérieur de la maison, comme son plan en général, sont fort peu renseignés par les fouilles ; on observe quelquefois les fondations, souvent légères, de murs de refend ou de cloisons à armature de bois, opérant la subdivision intérieure en plusieurs pièces, généralement deux ou trois, interprétées l'une comme la pièce à vivre, où se tient le foyer, et la ou les autres comme la chambre ou les annexes pour animaux et produits agricoles. La présence du foyer est implicite dans les actes qui n'en font à peu près aucune mention tandis que le terme de «cuisine », exceptionnel, est toujours lié à un grand établissement, monastère ou château. Synonyme de fonction domestique, le foyer n'est donc renseigné que par l'archéologie ou presque. La typologie de ces structures a donné lieu à d'importants travaux de synthèse (Pesez, 1998). En Normandie, la plupart correspondent à de simples foyers aménagés au sol, le plus souvent contre un mur de la pièce à vivre, plus rarement au centre. La sole est formée d'un lit d'argile recouvrant parfois une base de pierres ou de tessons. Deux cas au moins de cheminées murales ont toutefois été récemment observés en Normandie, à Jort et à Moult, au XIV siècle, où ont été mis au jour les piédroits encadrant l'âtre [ill. 6-7]. Il s'agit peut-être, à Moult, d'une cuisine aux murs chaulés. Des fours (à pain ?) sont parfois attestés, comme à Saint-Ursin (Hanusse, 2006, p. 86). 
Dans tous les cas, les actes notariés comme les fouilles confirment bien la partition ordinaire de la maison en deux pièces. Fin $\mathrm{XIV}^{\mathrm{e}}$-début $\mathrm{XV}^{\mathrm{e}}$ siècle, ces pièces sont volontiers louées à des occupants différents : Jouenne veuve Pierre Bellet, de Troarn, vend à Agnès, veuve Jehan Binete, « la moytié d'une maison avecquez la moytié du jardin » (f. 2ov). Ces moitiés de maisons sont baillées ou vendues avec une part équivalente des terres ou dépendances associées : Robine, veuve Renouf Le Roux, de Robehomme, vend à Monsieur Jehan Rouxel, recteur de cette paroisse, « une portion de maison avecquez la terre et court à icelle appartenant » (f. 37v). Tandis que l'emplacement des portes d'entrée est fréquemment visible à partir des dalles de seuil et parfois d'une crapaudine accueillant le pivot de la porte, les portes intérieurs sont plus discrets. La porte d'entrée est munie d'une serrure et d'une clé, comme à Varaville : « item les serreures des us » (f. 175), objets fréquemment mis au jour par les archéologues.

\section{Les annexes et dépendances}

Les parties habitables sont liées à un ensemble de biens et dépendances, tant intérieurs qu'extérieurs, terrains et meubles jouxtant ou meublant la maison qui, tous, participent de sa définition fonctionnelle et juridique. Un acte énumère ainsi, outre la chambre, une servitude de passage, une pièce en labour, deux huches pour ranger le linge et le pain, une « étable » ou plutôt bergerie : Rogière veuve Guillot Martine, de Bréville-les-Monts, fieffe une vergée de terre « et fut accordé que la dicte Rogière aira une cambre de la maison du dit Guillaume avecquez son yessue par devant et par derrière à toutes heures et tous le bas d'icelle cambre jusquez à la closture, elle aira lieu et place pour mectre demie acre de labour ; item deux huches ou solliers ; item estable pour héberger vint brebis sa vie durant » (f. 171v).

Tout comme en fouille, des cours attenantes à la maison sont bien attestées. Décaissées d'une cinquantaine de centimètres dans le sol, ces cours regroupent les activités domestiques, la bassecour... [ill. 8]. À Saint-Pair, un ensemble mesure « quarante piez de la court par devans la maison pour aler et venir au quemin du Roy » (f. 131v); à Démouville, on baille « la moitié d'une maison avec la moitié du jardin et sa part de la cour à icelle moitié appartenant » (f. 32v). La cour est parfois bornée, vraisemblablement par des pierres ou des poteaux : « la court si comme elle s'estend jusquez par les mectes mis par entre » (f. 175v). Le puits se situe généralement dans ou à proximité immédiate de la cour, comme à Moult [ill. 9-10]. À Escoville, deux frères se partagent une maison, chacun ayant son « aler et venir au pis (puits) du dit herbergage et de la court jusques par la moytié de la porte et de la cornière du gable (l'angle du pignon) de la neusve maison » (f. 98). On trouve aussi mentionnées la mare et la fosse à fumier (« mallière ») : « et la

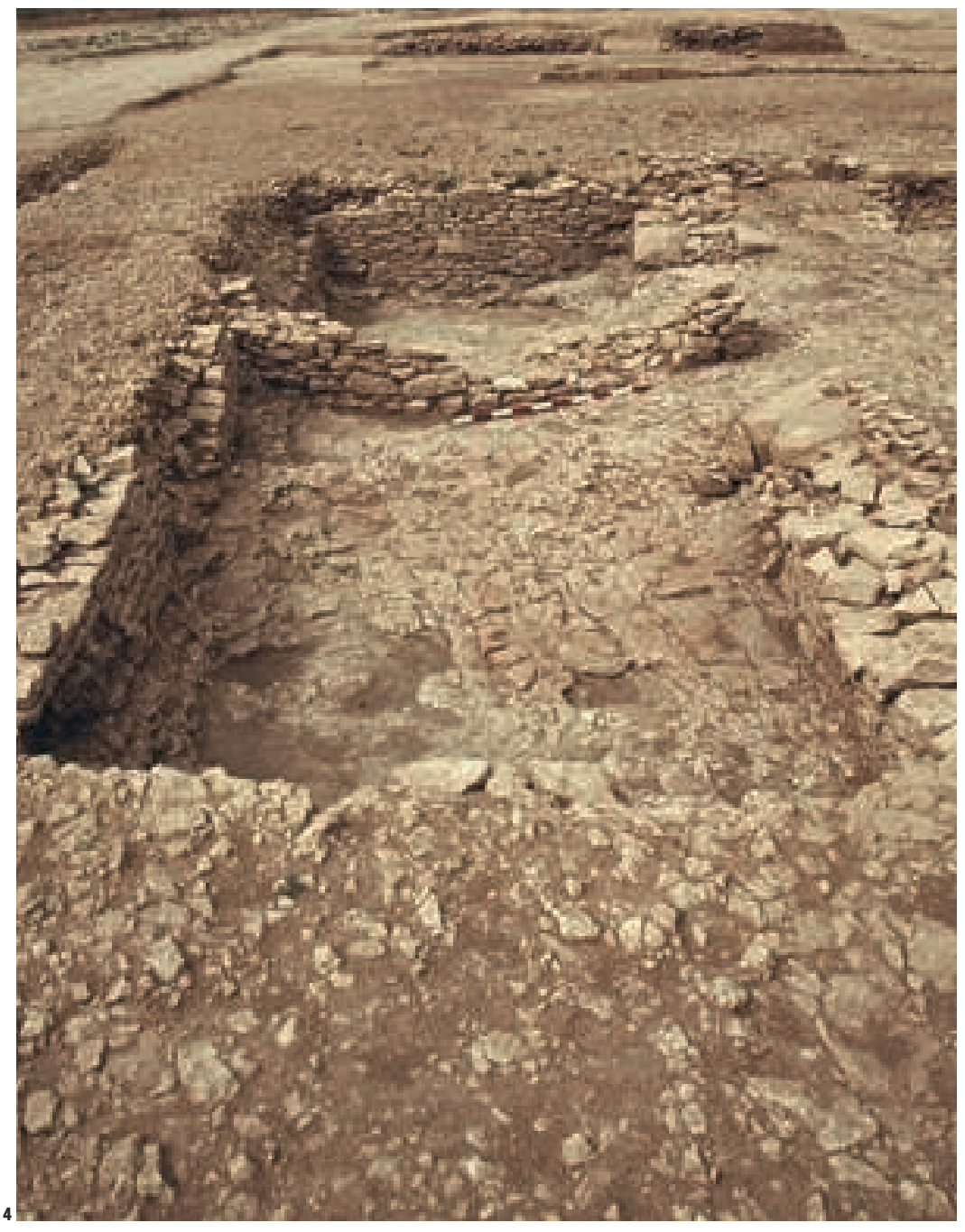

moytié de la mare pour faire sa mallière et son yessue à aler et venir à la dicte maison » (f. 105V).

Parmi les dépendances les plus courantes, on relève nombre d'étables, de granges ou de « carteries » (charretteries), parfois accolées entre elles : « un aménagement encontre le costé d'une granche audit Monsieur Jehan appartenant pour fère une estable» (f. 164). Une maison peut avoir plusieurs étables, comme à Varaville : « une petite maison où il a trois estables » (f. 24). Ici, hommes et animaux sont séparés ce qui n'est pas le cas partout en Normandie, y compris au sein d'un même « pays » comme la plaine de Caen où on a identifié des maisons mixtes à Trainecourt mais pas à Saint-Ursin (Hanusse, 2006, p. 88).

Le chemin menant à la grange, nécessaire aux charrois, est d'une importance manifeste :

« la granche n'aura que deux piés au bout que par devers la maison maignante (...) et si aura la dicte granche sa voie de six piés pour venir au quemin et se il estoit ainsy que un pommier qui est assis en droit la dicte granche fust nuysant à la dicte voie de six piez que ilz pourront prendre le quemin de l'autre part de la dicte granche » (f. 112). Plusieurs actes confirment la présence de petites forges 
4. Maisons à sols décaissés dans le substrat calcaire ou de plain-pied à Trainecourt, vues en enfilade.

5. Base supposée d'un escalier en bois contre l'un des murs gouttereaux d'un bâtiment de Moult, qui constituerait l'un des rares

témoignages archéologiques de maison à étage dans la Normandie de la fin du Moyen Âge. annexes des habitations, comme à Varaville « et si aura la pierre et le boys de la forge qui est endit herbergage » (f. 175). En revanche, les petites « constructions annexes » chères aux archéologues, cabanes, remises, appentis et autres poulaillers, ne sont pas ou très peu décrites ; il est une fois question d'un «buronet » (f. 38v). Ici encore, l'archéologie est le seul vecteur d'information sur ces annexes et notamment les caves et silos, bien présents sur la plupart des habitats [ill. 11].

\section{Le mobilier et l'équipement domestiques}

Un dernier chapitre de données concerne l'équipement domestique, au sujet duquel l'archéologie apporte une contribution limitée, le bois et autres matériaux organiques n'étant ordinairement pas conservés. On relève dans nos actes notariés des mentions récurrentes de meubles, lits, huches et coffres. Seuls les objets métalliques et la vaisselle la plus coûteuse sont évoqués, notamment les ustensiles à boire, de même que le linge de lit, draps, couettes, oreillers, ou le fil de couture. Colette, veuve Godeffroy, cède tous ses biens à l'hôpital de La Madeleinedes-Saulx, à Saint-Samson, où elle entend finir ses jours : « deux lis fournis, sept draps de liet, deux oreilliers, deux paelles d'erain (...) et tous ses nécessaires de boire » (f. 217). Un contrat de mariage énumère dans la maison des époux « six escuelles d'estain (...) huit draps de liet, tout le fil et couesses » (f. 170). Les outils d'artisans font l'objet de règlements spécifiques : « ils partageront par moitié tous leurs biens meubles, excepté tous les ostis du mestier de carpenterie et du pressour o tout son appareil (...) et si aira chacun son liet tel comme il a de présent et sa huche ou son coffre » (f. 30).

Cependant, bien que ténu, le catalogue des objets archéologiques, essentiellement façonnés en matériaux non périssables, complète et enrichit considérablement ces inventaires limités, on le voit à travers les quelques exemples qui précèdent, aux biens les plus coûteux. Le monnayage, les pratiques de l'écrit perceptibles à travers les sceaux, de même que le large spectre des activités quotidiennes, chasse et pêche, gestion des grains et autres denrées, élevage et boucherie, travail $\mathrm{du}$ bois et de la vigne, tous ces domaines ne sont que fort peu et très imparfaitement renseignés par les sources écrites. L'archéologie permet, de ce point de vue, de révéler de larges pans des invisibles de l'écrit. Archéozoologie, carpologie permettent en outre de documenter l'espace agraire et ses productions, essentielles.

Objet de recherche privilégié pour l'archéologue, la maison et ses dépendances sont logiquement au cœur de l'écrit régissant le quotidien à la fin du Moyen Âge. Selon un schéma relayé à la fois par les textes et les fouilles, cet habitat est fréquemment organisé en «fermes », au sens géographique

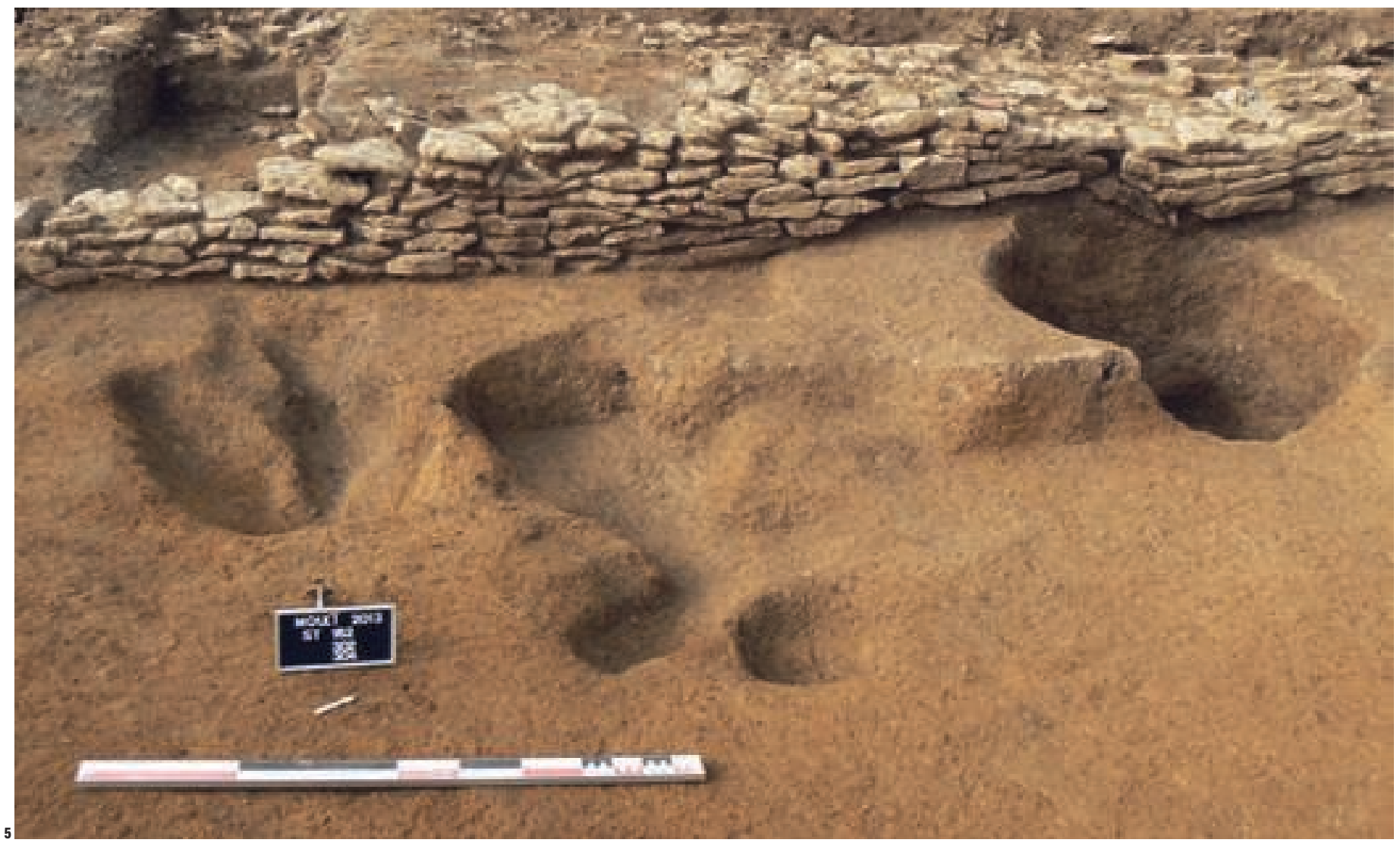



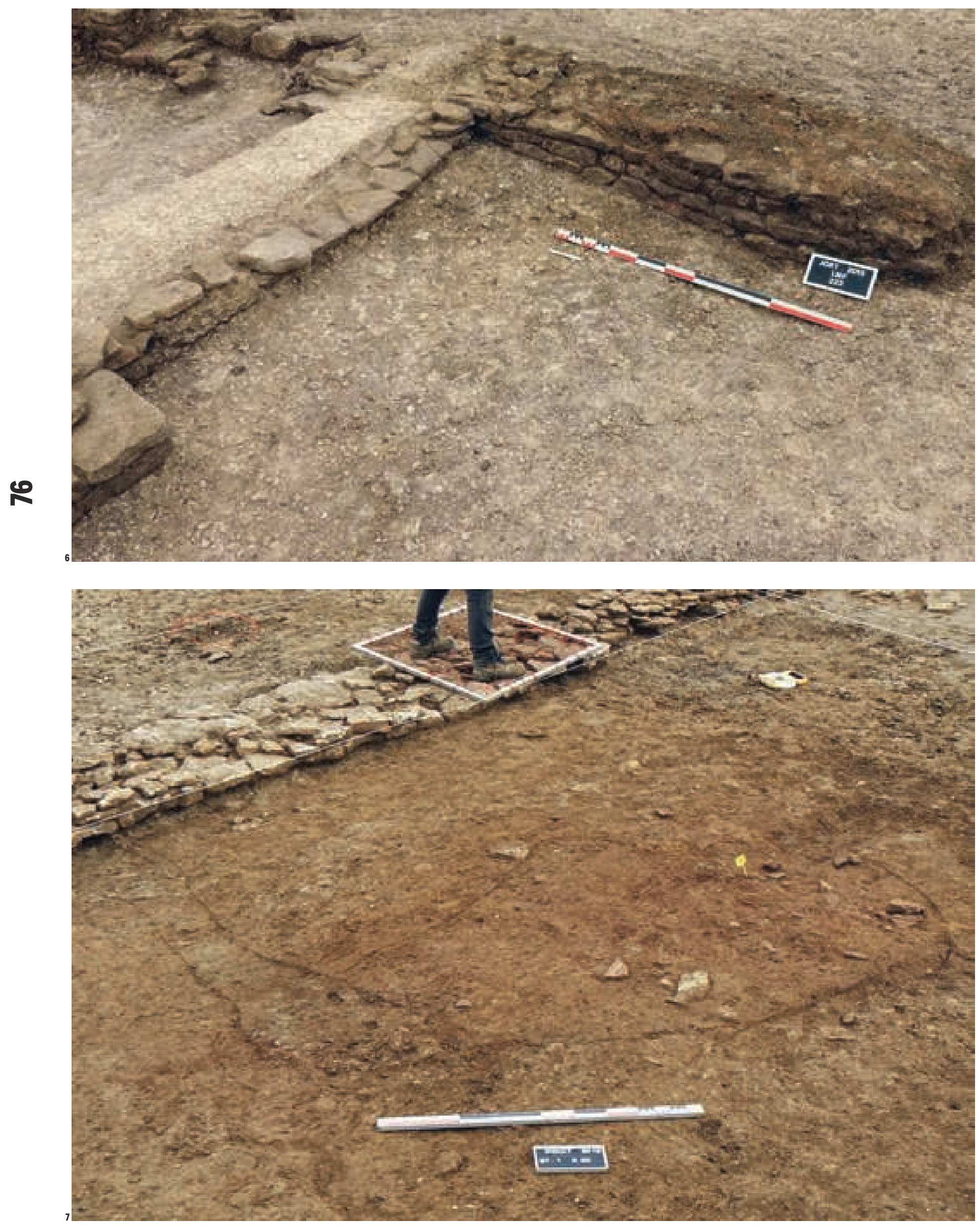


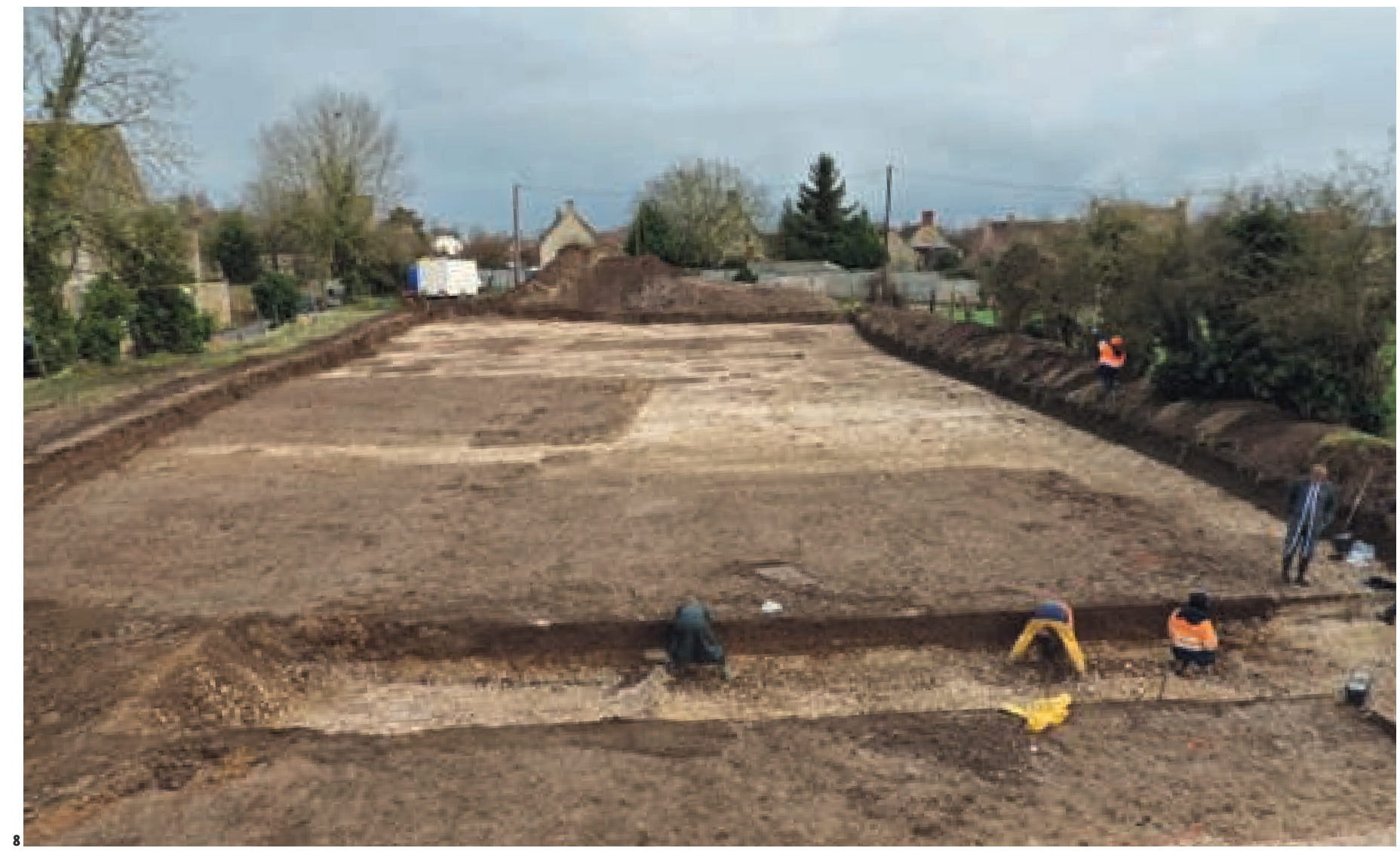

6. Âtre de cheminée murale dans la maison de Jort, délimité par un moellon

formant piédroit à gauche, et un mur en retour d'angle

à droite du cliché. Les pierres au fond de l'âtre sont rubéfiées par la chaleur.

7. Traces d'un âtre (zone rubéfiée) sur le sol d'une maison de Moult, contre l'un des murs gouttereaux, encadrées par deux saignées correspondant à l'emplacement des piédroits (ici à gauche). Des restes de carreaux en terre cuite et d'enduit de chaux ont été recueillis dans l'épaisseur de terre rubéfiée formée sur toute la largeur de l'âtre. 8. Au premier plan la cour, creusée sur une cinquantaine de centimètres, de la maison de Jort, en cours de fouille.

Un sondage permet de constater l'épaisseur de son remplissage. du terme : sièges de petite exploitation regroupant lieu de vie, dépendances économiques et espaces de production (Cabanne, 1984, p. 178). Ces fermes sont volontiers groupées en hameaux où l'on vit souvent sous le même toit. La définition de la maison, au bas Moyen Âge, inclut ses dépendances extérieures. En ce domaine, l'apport des fouilles s'avère essentiel, car les textes énumèrent seulement les grandes annexes, granges, « carteries », étables ou bergeries, forges. L'archéologie quant à elle révèle en partie ce que l'écrit tait: cheminées, caves, silos, petites constructions. Le recoupement de ces données, aussi lacunaires soient-elles, enrichit l'interprétation des découvertes archéologiques, en les replaçant dans un cadre plus large. Le site de Moult, par exemple, réunit plusieurs des composantes attestées et, dans une certaine mesure, explicitées par l'écrit : maison à étage, avec cheminée, cours et lopins séparés, clôtures et plantations, puits commun, voirie...

Plus largement, ces rapprochements participent de la « géographicité » croissante de l'habitatobjet de recherche en archéologie (Burnouf, 2008, p. 16-19, 48 sq. , 158-159). Les échelles d'investigation sont désormais plus vastes et pertinentes; il suffit pour s'en convaincre de comparer les plans des sondages des années 1970 avec ceux produits par l'archéologie préventive. Cet élargissement ouvre le questionnement sur des phénomènes dont l'ampleur dépasse l'échelon du site proprement dit, notamment sur l'impact des fléaux guerre-famine-épidémie, sans oublier le Petit âge glaciaire commençant, sur l'organisation des campagnes. Dans cet Occident qui, à la fin du XIV siècle, voit le repli global des cultures au profit des friches et couverts arborés (Duby, 1977, t. 2, p. 194 sq.), certaines évolutions ont de longue date été relevées par les historiens des textes, comme la diminution des baux de maisons ou l'augmentation des baux à parts, dans un marché foncier et immobilier étonnamment dynamique. Par ailleurs, comment se traduisent, dans l'habitat, les effroyables pics de mortalité dus à la Peste noire (« Hiroshima en Normandie », Bois, 1976, 200o)? Sur ces questions complexes, les données archéologiques sont probablement loin d'avoir encore tout dit : que penser par exemple, à Trainecourt, de la baisse notable du nombre et de la densité des unités d'habitat qui, à la charnière des XIII ${ }^{\mathrm{e}}$ et XIV ${ }^{\mathrm{e}}$ siècles, passent de 70 à moins de 10, avec une nette augmentation de leurs surfaces et du nombre de leurs annexes (Taupin, 1996) ? Sans doute ces mutations mériteraientelles aujourd'hui d'être davantage étudiées par les archéologues. 


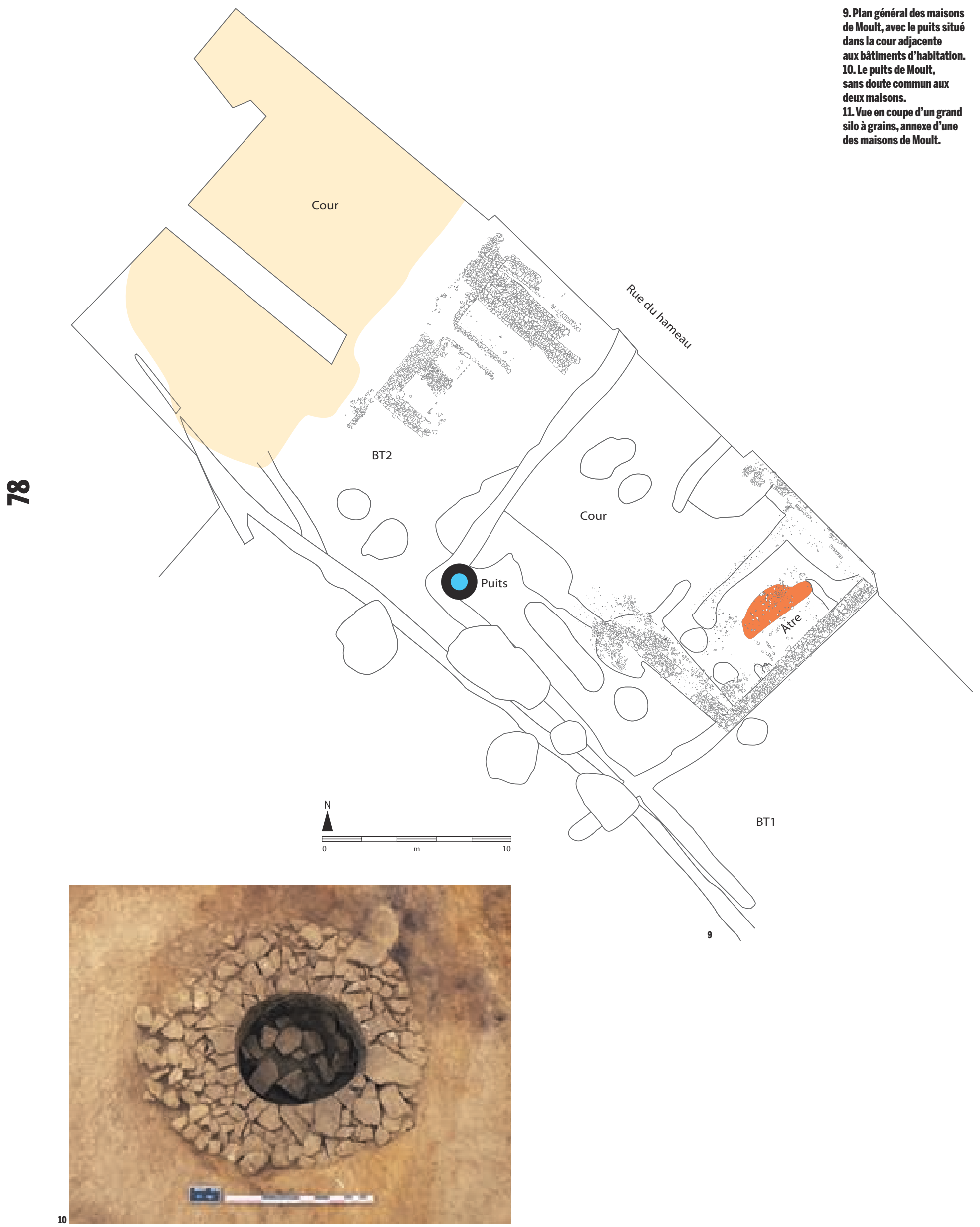




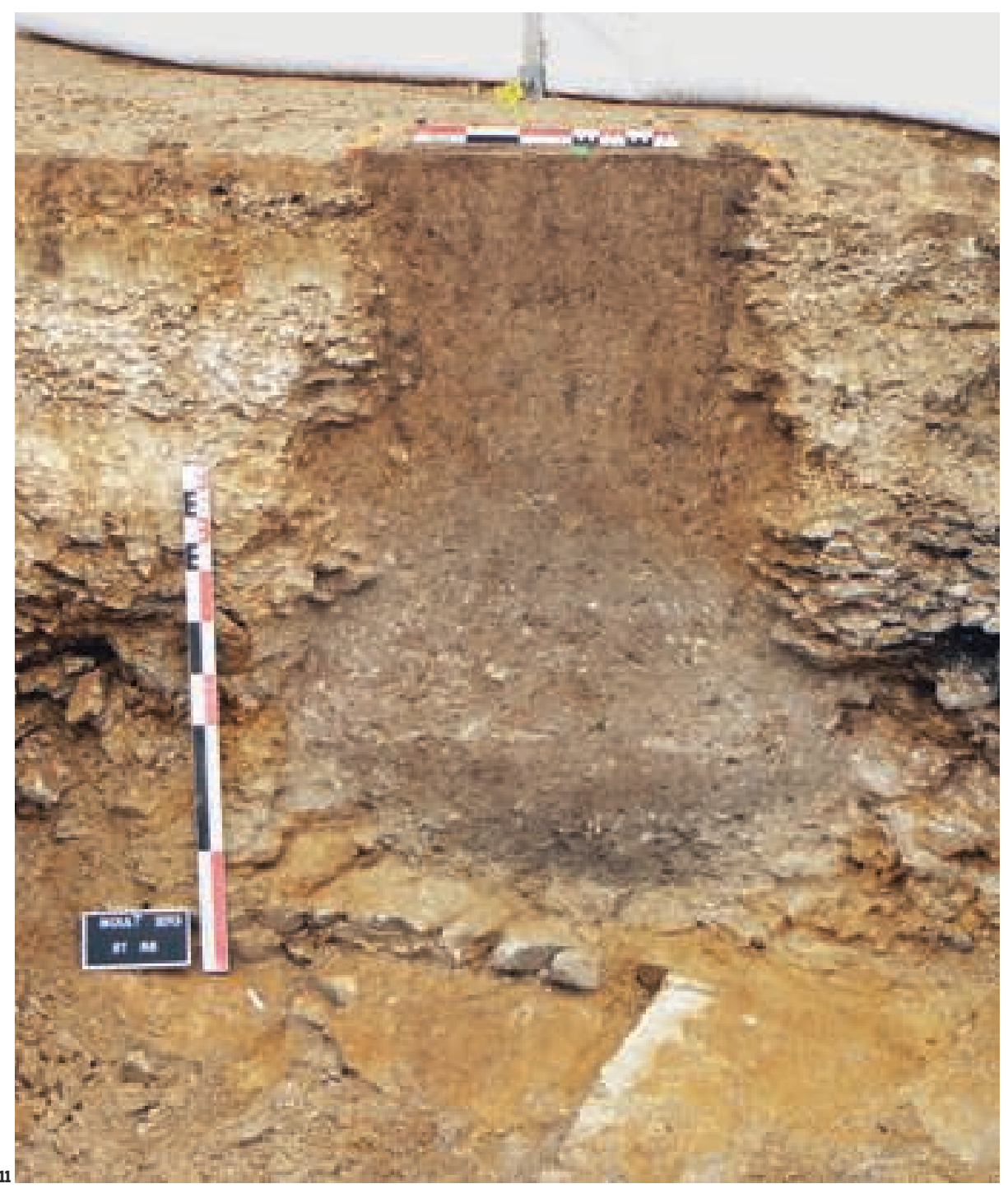

\section{Références bibliographiques}

MANeuVrier C. (éd.), 2010, Le registre de Guillaume Guérart, tabellion de Lisieux (1390-1393), Caen, Presses universitaires de Caen, « Enquêtes Rurales » 13, 344. p.

ANGERS D., 1994, « Une institution garante de l'ordre royal : le tabellionnage normand $\left(\mathrm{XIV}^{\mathrm{e}}-\mathrm{XV}^{\mathrm{e}}\right.$ siècles) $»$, Les Cahiers d'Histoire, XIV, 2, p. 5-22.

Bernard G., 1978, Guide des Archives départementales $d u$ Calvados, Caen, Archives départementales du Calvados, $411 \mathrm{p}$.

BoIs G., 1976, Crise du féodalisme : économie rurale et démographie en Normandie orientale, du début du $14^{e}$ au début du $16^{e}$ siècle, Paris, Éd. de l'École des hautes études en sciences sociales, « Cahiers de la Fondation nationale des sciences politiques » 202, $410 \mathrm{p}$.

BoIs G., 200o, La grande dépression médiévale, XIV et $X V^{e}$ siècles. Le précédent d'une crise systémique, Paris, Presses universitaires de France, $211 \mathrm{p}$.

Burnouf J., 2008, Archéologie médiévale en France. Le second Moyen Âge (XII ${ }^{e}-\mathrm{XVI}^{e}$ siècle), Paris, Inrap-La Découverte, $180 \mathrm{p}$.

CABANne C. (dir.), 1984, Lexique de géographie humaine et économique, Paris, Dalloz, $432 \mathrm{p}$

Carpentier V., 2015, Moult (Calvados), «le Val des Cigognes ", Un hameau des XIII'-XV $V^{e}$ siècles aux portes du pays d'Auge, Rapport d'opération, InrapSRA Normandie, $164 \mathrm{p}$
Carpentier V. (dir.), 2017, Jort (Calvados), «le Moulin fouleux », B696; Au coeur d'un village: Vestiges de l'Antiquité à 1944 au bord de la Dives, Rapport d'opération, Inrap-SRA Normandie, 334 p.

Duby G., 1977, L'économie rurale et la vie des campagnes dans l'Occident médiéval, Paris, Flammarion, «Champs », 2 vol., 285 et 288 p.

ÉCOle PRATIQUe Des Hautes ÉTUdes, 1970, Archéologie du village déserté, Paris, A. Colin, 2 vol.

Hanusse C., 20o6, « L'organisation des espaces de circulation dans l'habitat rural de la plaine de Caen. L'exemple du site des "Fosses Saint-Ursin" (XIII ${ }^{\mathrm{e}}$ $\mathrm{XIV}^{\mathrm{e}} \mathrm{s}$.) », in Alexandre-Bidon D., Piponnier F., Poisson J.-M. (dir.), Cadre de vie et manières d'habiter (XII $-X V I^{e}$ siècle), Actes du $8^{e}$ Congrès international de la Société d'archéologie médiévale (Paris, 11-13 octobre 2001), Caen, Publications du CRAHM, p. 81-89.

HANusse C., JARRY T., 2007, « Espace bâti et habitation en Normandie (Plaine de Caen) du XIII ${ }^{\mathrm{e}}$ au Xv ${ }^{\mathrm{e}}$ siècle. Mise en regard des sources archéologiques et textuelles », in MAdeline P., MoriceAu J.-M. (éd.), Bâtir dans les campagnes. Les enjeux de la construction de la Protohistoire au XXI siècle, Caen, Pôle rural MRSH-Caen, « Bibliothèque du pôle rural » 1, p. 133-152

Hincker V., Thiron D., Maneuvrier C., 1999, Déviation de Vieux-Fumé R.D.4o, Rapport d'opération, Conseil général du Calvados, t. 2, $140 \mathrm{p}$.

LASSURE C., 1985, « Le traitement de la bibliographie dans le volume Bretagne du corpus de l'architecture rurale française », L'architecture vernaculaire, IX, p. 92.
Le LORIER L., 1911, Notes sur un registre du tabellionnage d'Argences-Troarn-Varaville de la fin du XIV siècle conservé aux Archives du Calvados, Paris, Imprimerie nationale, $20 \mathrm{p}$.

LORREN C., 1998, « Normandie. 18. Grentheville (Calvados). Trainecourt. XIII ${ }^{\mathrm{e}}$ siècle », in PEsEz J.-M., Esquieu Y. (dir.), Cent maisons médiévales en France (du XII au milieu du XVI ${ }^{e}$ siècle): un corpus et une esquisse, Paris, CNRS éd., "Monographie du CRA » 2o, p. 205-206.

LORREN C., 2002, « Dans quelle mesure l'archéologie offre-t-elle une image fidèle de la vie au village à la fin du Moyen Âge? Quelques observations sommaires à propos de fouilles récentes dans la Plaine de Caen », in Berthelot S., Marin J.-Y., Rey-Delqué M. (dir.), Vivre au Moyen Âge. Archéologie du quotidien en Normandie, XIII ${ }^{e}-X V^{e}$ siècles, Milano, 5 Continents, p. 100-109.

Pesez J.-M., 1998, « Le chauffage : foyers et cheminées », in Pesez J.-M. Esquieu Y. (dir.), Cent maisons médiévales en France (du XII ${ }^{e}$ au milieu du $X V I^{e}$ siècle): un corpus et une esquisse, Paris, CNRS éd., « Monographie du CRA » 20, p. 109-113.

SAUVAGE R.-N., 1911, L'abbaye de Saint-Martin-deTroarn, au diocèse de Bayeux, des origines à la fin $d u$ seizième siècle, Caen, L. Jouan, « Mémoires de la Société des antiquaires de Normandie », t. XXXIV, LII-524 p.

Taupin M.-C., 1996, « Hameau de Trainecourt XIII ${ }^{\text {- }}$ $\mathrm{xV}^{\mathrm{e}}$ s. », Ruralia, I, Památky archeologické, Suppl. 5, Prague, p. 211-216. 